Research Paper

\title{
Macrophage Activation Syndrome-Associated Markers in Severe Dengue
}

\author{
Hasliana Azrah Ab-Rahman¹,2, Hafiz Rahim4, Sazaly AbuBakar ${ }^{1,2}$, Pooi-Fong Wong ${ }^{3 凶}$ \\ 1. Tropical Infectious Disease Research \& Education Centre (TIDREC), Faculty of Medicine, University of Malaya, 50603 Kuala Lumpur, Malaysia; \\ 2. Department of Medical Microbiology, Faculty of Medicine, University of Malaya, 50603 Kuala Lumpur, Malaysia; \\ 3. Department of Pharmacology, Faculty of Medicine, University of Malaya, 50603 Kuala Lumpur, Malaysia; \\ 4. Department of Medicine, Faculty of Medicine, University of Malaya, 50603 Kuala Lumpur, Malaysia. \\ $\bowtie$ Corresponding author: Dr. Wong Pooi-Fong (Ph.D), wongpf@um.edu.my Tel.: +603-7967 7022 ext 2065; fax: +603-7967 4791.
}

(1) Ivyspring International Publisher. Reproduction is permitted for personal, noncommercial use, provided that the article is in whole, unmodified, and properly cited. See http://ivyspring.com/terms for terms and conditions.

Received: 2015.08.28; Accepted: 2016.01.05; Published: 2016.02.17

\begin{abstract}
Hemophagocytosis, a phenomenon of which activated macrophages phagocytosed hematopoietic elements was reportedly observed in severe dengue patients. In the present study, we investigated whether markers of macrophage activation syndrome (MAS) can be used as differential diagnostic markers of severe dengue. Two hundred and eight confirmed dengue patients were recruited for the study. Sandwich ELISA was used to determine serum ferritin, soluble CD163 (sCD163), and soluble CD25 (sCD25) levels. The population of circulating CD163 (mCD163) monocytes was determined using flow cytometry. Receiver operating characteristic (ROC) analysis was plotted to determine the predictive validity of the biomarkers. Serum ferritin and sCD163 were found significantly increased in severe dengue patients compared to dengue fever patients $(P=0.003)$. A fair area under ROC curves (AUC) at 0.72 with a significant $P$ value of 0.004 was observed for sCD163. sCD25 and mCD163 levels were not significantly different between severe dengue and dengue fever patients. Our findings suggest that in addition to serum ferritin, sCD163 can differentiate severe dengue from that of dengue fever patients. Hence, sCD163 level can be considered for use as a predictive marker for impending severe dengue.
\end{abstract}

Key words: Hemophagocytic lymphohistiocytosis, macrophage hyperactivation, cytokine storm, CD163, CD25.

\section{Introduction}

Dengue is a mosquito-borne disease caused by dengue viruses from the Flaviviridae family $[1,2]$. It is a disease affecting an estimated 3.97 billion people in over 128 endemic countries, including Malaysia [3]. It is described as a dynamic febrile illness which can manifests as self-limiting mild fever or the more severe life-threatening forms with presentation of plasma leakage, hemorrhage or severe multi-organ impairments. A number of mechanisms have been forwarded to explain the pathogenesis of severe dengue, including antibody-dependent enhancement (ADE) of virus infection [4,5], overwhelming memory $\mathrm{T}$ cell activation [6] and exaggerated pro-inflammatory cytokine responses $[7,8]$ leading to the more severe or fatal dengue hemorrhagic fever (DHF) and dengue shock syndrome (DSS) [9-11].

In recent decades, an uncommon phenomenon of macrophage activation syndrome (MAS) or hemophagocytic syndrome (HS) is increasingly reported in patients with severe dengue. Hemophagocytosis was reported in dengue with multi-organ complications [12], observed in severe dengue involving both children and adults [13-15] and associated with dyserythropoiesis [16]. MAS is a severe systemic inflammatory condition due to excessive activation and proliferation of $\mathrm{T}$ cells and well-differentiated macrophages. The hyperactivated but dysregulated immune responses lead to overwhelming inflammatory responses resulting in non-remitting high fever, hepatomegaly and splenomegaly, lymphadenopathy, haemorrhage and central nervous system dysfunction [17]. Diagnostic features of macrophage activation syndrome include hyperferritinemia (above 10000 $\mu \mathrm{g} / \mathrm{L})$, cytopenia, coagulopathy, abnormal liver func- 
tion tests, hypertriglyceridemia, hypoalbuminemia, hyponatremia, hemophagocytosis and elevated serum sCD25 and sCD16 levels [17-21]. Complications associated with MAS can be fatal. It is the most common underlying presentation of systemic juvenile idiopathic arthritis (sJIA) [22-24]. Presence of hemophagocytosis activity and increased CD163 staining of the bone marrow are among some of the histopathological features of MAS and the best evidence for disease prognosis [25]. There have been several reported cases of hemophagocytosis in dengue with extremely high serum ferritin level [12, 14, 26, 27]. Markedly elevated level of ferritin has been used as a clinical biomarker for severe dengue, and is considered as one of the most important serum biomarker for dengue disease $[28,29]$. Meanwhile, CD163 is an important macrophage activation marker as its expression and secretion was reported in several infectious diseases including malaria, hepatitis C and HIV [30-35] but there was no previous report on dengue.

Given the close resemblance of a number of clinical features of MAS and severe dengue [26], and the observation of hemophagocytosis activity in the bone marrow in dengue cases [13, 14, 16, 27], further understanding of the relationship between MAS and dengue immunopathogenesis especially in severe dengue disease is warranted. This is the first study that examined the levels of important biomarkers of MAS in patients with dengue fever and severe dengue.

\section{Materials and methods}

\section{Study Approval}

This study was approved by the Medical Ethics Committee of the University Malaya Medical Centre (Ethics No. 908.9). Written informed consent was obtained from patients to authorize their participation in the study. This study conformed to the Declaration of Helsinki and Malaysian Good Clinical Practice (GCP) guidelines. Blood specimens were obtained from recruited adult patients seen at the infectious disease ward (ID ward) of University of Malaya Medical Centre (UMMC), following 2 to 7 days of fever between year 2012 and 2013. Two hundred eight patients' clinically- and laboratory-confirmed with dengue were recruited. Confirmation of dengue was based on the detection of dengue specific IgM antibody or dengue non-structural protein 1 (NS1) antigen or dengue specific IgG antibody. Clinical diagnosis of the disease was based on the 2009 World Health Organization (WHO) revised dengue classification. Sera of 48 healthy adult volunteers served as healthy control. Blood specimens were obtained into two vacutainer tubes (BD Biosciences, USA). Blood were collected into EDTA-containing tube for immunostaining while serum separator tube (SST) was used for cytokine assays, as well as for NS1 antigen, dengue IgM and dengue IgG detection. Blood from SST tube was initially spun at $1200 x g$ for 10 minutes. Sera were harvested and kept in $-80^{\circ} \mathrm{C}$, until needed.

\section{Detection of Dengue IgM, IgG Antibodies and NS1 Antigen}

Dengue specific IgM antibody and IgG antibody detection was performed using ELISA (SD Standard Diagnostics Inc., Korea) and dengue NS1 antigen detection was performed using the Dengue NS1 Antigen Strip (Bio-Rad, Hercules, California).

\section{Membrane-Bound CD163 (mCD163) Im- munostaining}

EDTA blood $(200 \mu \mathrm{L})$ was used for immunostaining. Whole blood was stained with $10 \mu \mathrm{L}$ of both mouse anti-human CD14 FITC (clone M $\phi \mathrm{P9}$ ) and mouse anti-human CD163 PE (clone GH1/61) (Becton Dickinston, USA) and incubated for 30 minutes in the dark at room temperature. The stained whole blood was lysed with $2 \mathrm{~mL}$ of FACS lysing buffer (Becton Dickinston, USA). The lysed blood was then spun at 500xg for 4 minutes. The supernatant was discarded and the pellet was washed with $2 \mathrm{~mL}$ of FACS buffer containing 1xPBS, $2 \%$ foetal bovine serum and sodium azide ( ${ }^{\text {st }}$ Base, Singapore; Gibco, USA; Sigma Aldrich, USA) [36]. The pellet was re-suspended with FACS buffer prior for analysis with FACS Canto II (Becton Dickinston, USA). Samples acquisition from FACS Canto II was performed following the manufacturer's protocol with only minor modifications [37]. Data were analysed using BD FACSDiva version 6.1.2 software (BD Bioscience, USA).

\section{Quantitation of Soluble Serum Biomarkers}

Ferritin quantitation was done at the Clinical Diagnostic Laboratory (CDL) of University Malaya Medical Center (UMMC) based on chemiluminescence enzyme immunoassay (CEIA) using ADVIA Centaur $^{\circledR}$ Immunoassay System (Siemens Healthcare Diagnostics, USA). Soluble serum CD163 and CD25 were determined by using ELISA (R\&D System, Minneapolis), following manufacturers' protocol with minor modifications. Mouse anti-human CD163 and CD25 antibody at concentration $2.0 \mu \mathrm{g} / \mathrm{mL}$ were immobilised in 96-well plate in coating buffer $(0.05 \mathrm{M}$ carbonate-bicarbonate, $\mathrm{pH}$ 9.6), blocked with blocking buffer (50mM tris, $0.14 \mathrm{M} \mathrm{NaCl}, 1 \% \mathrm{BSA}, \mathrm{pH} 8.0$ ), and then washed with phosphate buffered saline with $0.05 \%$ tween-20. Color was developed by adding TMB substrate solution into each well and incubated for 5 
minutes for sCD163 and 20 minutes for sCD25 in dark at room temperature. Reaction was stopped by adding $2 \mathrm{M} \mathrm{H}_{2} \mathrm{SO}_{4}$ and the plate was read at wavelength $450 \mathrm{~nm}$ with correction at $570 \mathrm{~nm}$. A 4-parameter logistic standard curve was generated from the optical density (OD) readings. The detection limit of each soluble protein was as follows: $1.56 \mathrm{ng} / \mathrm{mL}$ (sCD163) and $31.25 \mathrm{pg} / \mathrm{mL}$ (sCD25).

\section{Statistics}

One-way ANOVA test with Bonferroni's multiple comparison was performed using GraphPad Prism version 5.01 software. Receiver operating characteristic (ROC) was plotted to determine the predictive validity of a biomarker to forecast impending severe dengue. The value of the area under the ROC curve (AUCs) was calculated to determine the robustness of the test. The AUCs of $0.5-0.69$ was considered as poor, $0.7-0.79$ as fair, and $0.8-0.89$ as good, 0.9-0.99 as excellent and 1.0 as perfect. Data were presented as median (mean \pm standard error) and percentage, where applicable. A $P$-value lower than 0.05 was considered significant.

\section{Results}

\section{Confirmation and Classification of Dengue Cases}

A total of 208 patients were recruited for the present study. Clinical and laboratory profiles of the patients were shown in Table 1 . There were 20 confirmed severe dengue patients and 188 dengue fever patients determined based on their anti-dengue IgM, dengue antigen NS1 or anti-dengue IgG status. The laboratory confirmation for dengue was performed according to the Centers for Disease Control and Prevention (CDC) guideline (Fig. 1) [38]. The classification of severe dengue and dengue fever was based on the 2009 WHO revised classification. In brief, dengue fever can present as a mild self-limiting fever or as the more severe forms of the disease with the observation of severe plasma leakage or severe bleeding and multiple organ failures [39]. In the present study, the severe dengue group consisted of four patients with plasma leakage, twelve patients had severe organ impairments, one had plasma leakage with organ impairment, one developed plasma leakage with hemorrhage, and two patients had organ impairments and hemorrhage. The control group consisted of 61 healthy volunteers, 48 were serologically negative for dengue $\operatorname{IgG}$ whereas the remaining had positive dengue IgG.

\section{Serum Levels of Soluble Ferritin, sCD163 and sCD25 in Severe Dengue Patients}

The concentrations of the three distinct markers of MAS were shown in Figure 2, Serum ferritin was significantly elevated in severe dengue patients when compared to the dengue fever patients and healthy volunteers with median values of $15172.00 \mu \mathrm{g} / \mathrm{L}$ $(25758.00 \mu \mathrm{g} / \mathrm{L} \pm 6857.88)$ in severe dengue, 4026.50 $\mu \mathrm{g} / \mathrm{L}(8877.71 \mu \mathrm{g} / \mathrm{L} \pm 1730.37)$ in dengue fever and $57.0 \mu \mathrm{g} / \mathrm{L}(82.97 \mu \mathrm{g} / \mathrm{L} \pm 11.20)$ in healthy volunteers (Fig. 2A). The level of sCD163 was significantly higher in the severe dengue group, with a median value of $399.50 \mathrm{ng} / \mathrm{mL}(781.13 \mathrm{ng} / \mathrm{mL} \pm 254.26)$ compared to $192.36 \mathrm{ng} / \mathrm{mL}(338.88 \mathrm{ng} / \mathrm{mL} \pm 42.03)$ in the dengue fever group and $226.01 \mathrm{ng} / \mathrm{mL}(237.54 \mathrm{ng} / \mathrm{mL} \pm 14.67)$ in healthy volunteers (Fig. 2B). Significant elevation of sCD25 was observed in the severe dengue group compared to the healthy control group but not with the dengue fever group. The median values sCD25 level were $1490.00 \mathrm{pg} / \mathrm{mL}(2663.26 \mathrm{pg} / \mathrm{mL} \pm 520.55)$ in the severe dengue group, $1400.00 \mathrm{pg} / \mathrm{mL}$ (1961.52 $\mathrm{pg} / \mathrm{mL} \pm 230.82)$ in the dengue fever group and 221.81 $\mathrm{pg} / \mathrm{mL}(228.49 \mathrm{pg} / \mathrm{mL} \pm 11.79)$ in healthy volunteers (Fig. 2C). In addition, the correlation between the serum level of sCD163 and ferritin among severe dengue patients was examined. A significant correlation was observed between the level of sCD163 and ferritin with correlation coefficient $(\mathrm{r}$ ) value of 0.72 (Fig. 3A). A fair and perfect area under ROC curves (AUC) at 0.72 and 1.00 were observed when the level of sCD163 of the severe dengue group was compared to the dengue fever group (Fig. 3B) and against ferritin among severe dengue group, respectively (Fig. 3C). This suggests that sCD163 level was directly associated with manifestations of dengue disease and its severity.

TABLE 1. Clinical and laboratory profiles and complications observed among dengue patients.

\begin{tabular}{|c|c|c|c|c|c|}
\hline \multirow[t]{2}{*}{ Dengue Disease } & \multirow{2}{*}{$\begin{array}{l}\text { Enrolled } \\
\text { Patients }\end{array}$} & \multicolumn{3}{|c|}{ Laboratory Confirmation of Dengue Disease } & \multirow[t]{2}{*}{${ }^{*}$ Complications } \\
\hline & & IgM Status & NS1 Antigen & IgG Status & \\
\hline Severe Dengue & $9.6 \%(20)$ & Positive (14) & Positive (10) & Positive (1) & $\begin{array}{l}\text { Plasma Leakage (6); Hepatitis (15); Compromising Respiratory (1); Confused } \\
\text { CNS (1); Severe Hemorrhage (2) }\end{array}$ \\
\hline Dengue Fever & $90.4 \%(188)$ & Positive (88) & Positive (63) & Positive (37) & $\begin{array}{l}\text { Vomiting (63); Abdominal Pain (47); Nausea (2); Diarrhea (6); Mucosal } \\
\text { Bleeding (8); PV Bleeding (1); Epigastric Pain (1); Gum Bleeding (5); Epistaxis } \\
\text { (3); Unspecified Conditions (52) }\end{array}$ \\
\hline
\end{tabular}

*Certain patients had more than one complication. 




Figure 1. Procedural workflow used for the confirmation of dengue.
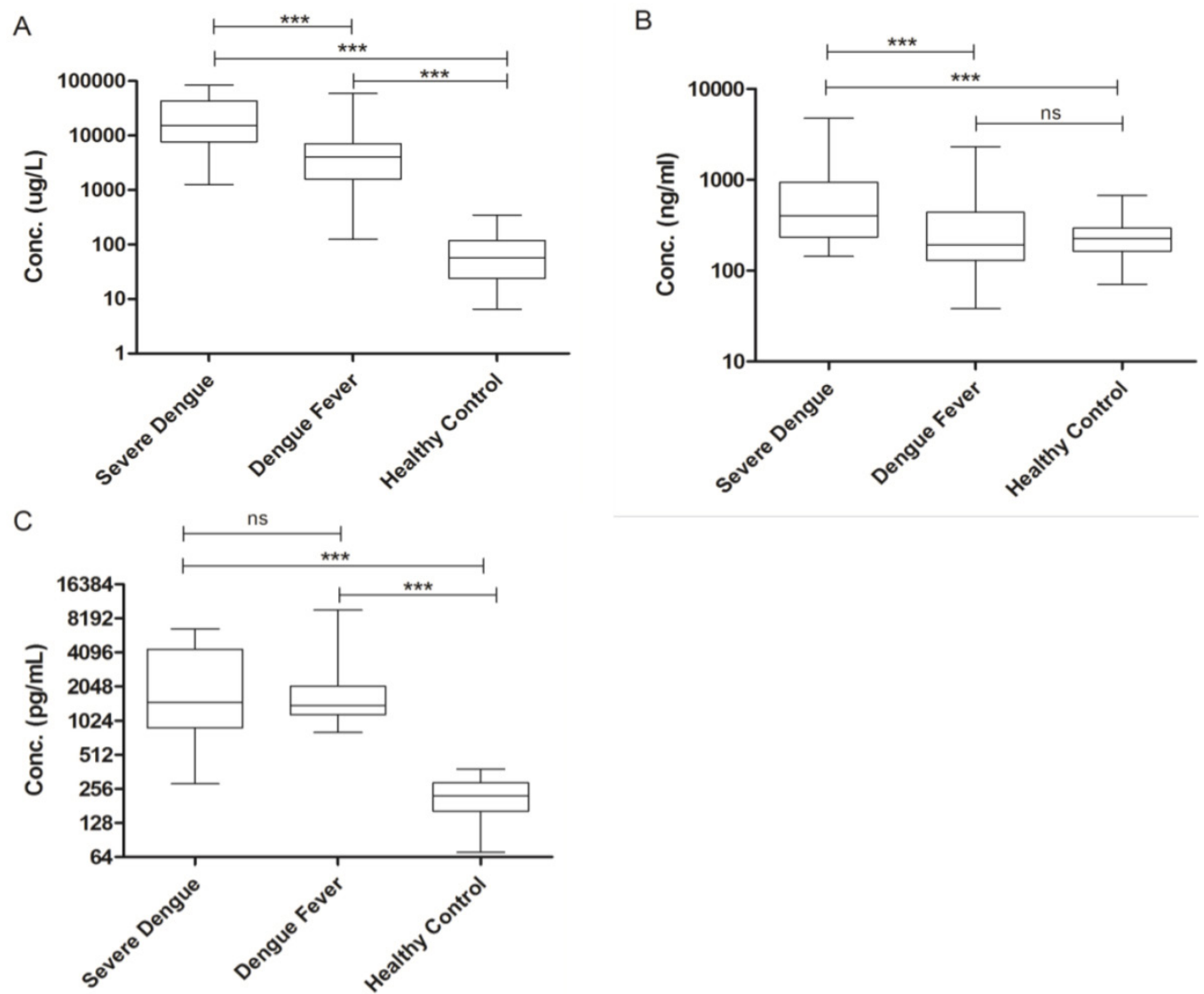

Figure 2. Soluble Serum Biomarkers in Dengue. (A) Ferritin, (B) $s C D 163$, (C) $s C D 25$. One-way ANOVA test with Bonferroni's multiple comparison was performed using GraphPad Prism version 5.01 software. $* P<0.05$, $* * P<0.01$, ***P $<0.001$, ns indicates non-significant. 
A

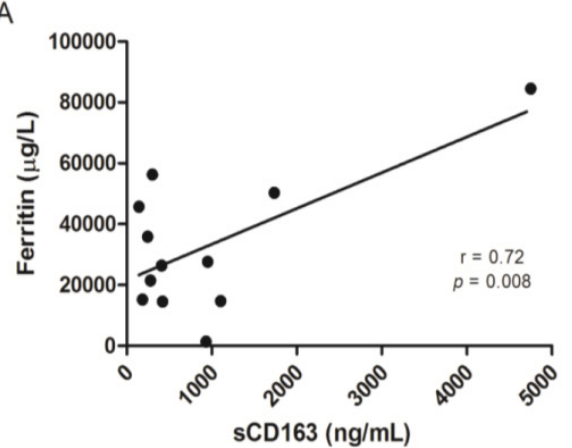

C

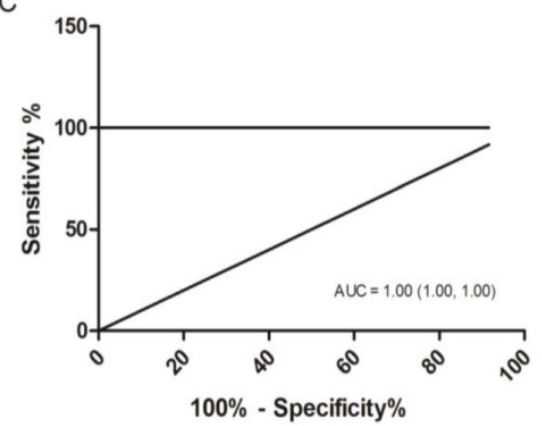

B

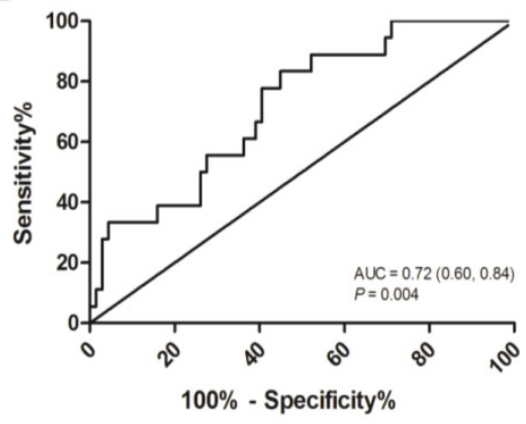

Figure 3. Correlation analysis and the receiver operating characteristic (ROC) curves of $s C D 163$. (A) Correlation of serum ferritin to $s C D 163$ in severe dengue patients, $P=0.008$. (B) ROC curve for $s C D 163$ in severe dengue group in comparison to the dengue fever group. $A$ fair area under ROC curves (AUC) at 0.72 was obtained. (C) ROC curve combining sCD163 and ferritin above than $10000 \mu \mathrm{g} / \mathrm{L}$ in the severe dengue group. A perfect AUC score at 1.00 was obtained.

\section{Flow Cytometry Immunostaining of Circulat- ing Membrane-Bound CD163 Monocytes}

CD163 receptor is shed from macrophages and accumulates in the plasma as soluble form upon inflammatory stimulation. To determine the total number of circulating CD163-bearing monocytes in dengue patients, CD163 cells were gated from the total monocytes population (Fig. 4A). Approximately $81.9 \%(80.9 \% \pm 1.15)$ of the total monocytes from the healthy control group was positive for CD163 with $51.9 \%(53.8 \% \pm 3.84) ; 55.6 \%(53.6 \% \pm 3.10)$ for the severe dengue and dengue fever group (Fig. 4B), respectively. This suggests that in general, there is an overall reduction in the population of CD163 monocytes in dengue infection.

\section{Discussion}

Dengue virus infection can results in severe disease that if not treated early can cause deaths. It is currently among the most important mosquito-borne disease worldwide. Presently, there is no specific drug for dengue treatment or an approved vaccine to prevent dengue. Dengue patients are usually treated in the hospital by close monitoring and good supportive care. Most dengue patients recover uneventfully within 5 days following onset of fever. Only less than $10 \%$ would develop severe dengue which manifests with severe intravascular leakages, severe organ impairments and severe heamorrhage. To date, there are no specific ways of determining which among the dengue patients would develop severe dengue. A long list of soluble markers which were observed to be elevated in severe dengue patients has been described. Severe dengue tends to present with high levels of cytokines such as IFN- $\gamma$, TNF- $\alpha$, IL1- $\beta$, IL-2, IL-6, IL-8, and IL-10 [40-44]. Moreover, high level of TGF- $\beta$ [9], histamine [44, 45, 46], IL-13, IL-7, GM-CSF $[48,49]$ have also been described. More recently, extremely high level of serum ferritin was reported in severe dengue patients [28, 29]. The level of serum ferritin was significantly different in patients with severe dengue with plasma leakage, haemorrhage and shock [29].

Hyperferritinemia, specifically above 10000 $\mu \mathrm{g} / \mathrm{L}$, elevated sCD163 and sCD25 levels are the few laboratory diagnostic features of macrophage activation syndrome (MAS) $[24,38,50]$. Results from the present study suggest that high serum ferritin and sCD163 levels occurred in patients with severe dengue. sCD163, in particular, can robustly differentiate severe dengue patients from those with only dengue fever. CD163 found on macrophages is a haemoglobin scavenger receptor and has been widely described to be involved in the clearance of haemoglobin to prevent oxidative stress [51,52]. It mediates the internalization of haemoglobin-haptoglobin ( $\mathrm{Hb}-\mathrm{Hp})$ complexes and sheds its extracellular part into plasma [53]. It is known that dengue virus infection produces pro-inflammatory cytokines such as TNF- $\alpha$, IFN- $\gamma$ 
and IL-6 that can further induce the production of important anti-inflammatory cytokines especially IL-10 [8, 54, 55] known to activate monocytes/macrophages that express CD163 [53-59]. The elevated level of sCD163 reflects activation of monocytes/macrophages consistent with MAS in dengue.

Membrane bound CD163 (mCD163) on the other hand, is solely expressed on cells of monocyte-macrophage lineage. The membrane bound CD163 is shed from cells and accumulates in the plasma as soluble form upon exposure to inflammatory stimuli. The expression of mCD163 is inversely correlated to its soluble form. In agreement with a previous report [60], increased in SCD163 level in severe dengue patients is inversely correlated to the percentage of monocytes/macrophages expressing mCD163. The percentage of positive mCD163 cells however, was not significantly different between severe dengue and dengue fever patients but was reduced in comparison to healthy controls. Since reduced number of cells expressing mCD163 was also observed in acute dengue patients, this suggests that active shedding of mCD163 takes place even in dengue fever, perhaps to dampen inflammation caused by the infection. Increased level of sCD163 however, is not unique to dengue as it is noted in critically ill patients whom have contracted various inflammatory diseases [61]. Nonetheless up-regulation of CD163 is specific to macrophage adaptation towards inflammation shown in hemophagocytosis in bone marrow, independent of underlying diseases [62].

In addition to sCD163, sCD25 is secreted by activated T cells. In our study, sCD25 level did not sig-
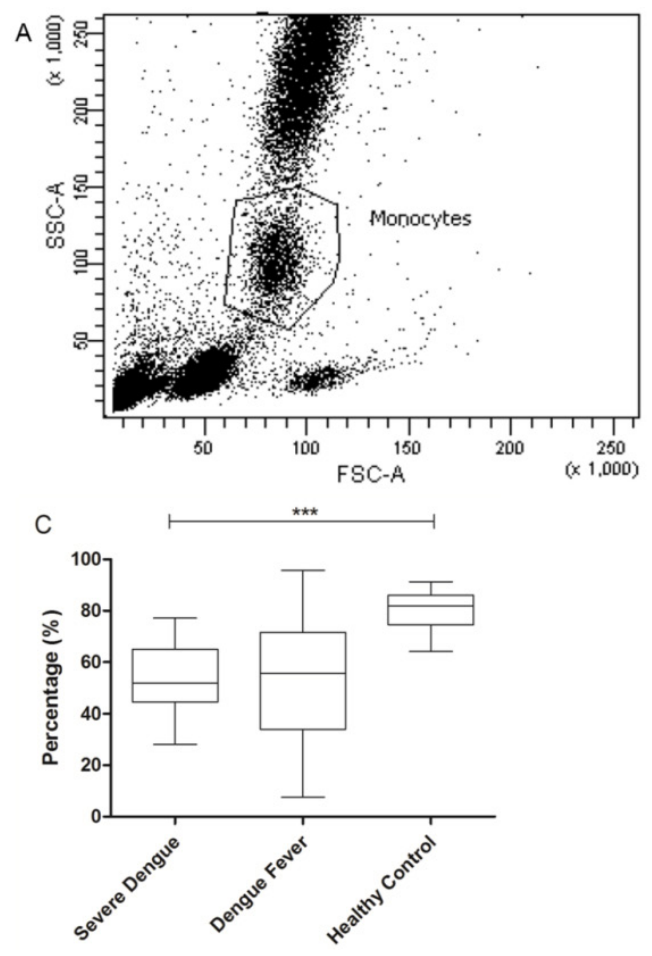

nificantly differ between severe dengue patients from those of dengue fever patients. There was a significant difference however, between acute and convalescence phase sera consistent with heightened activation of $\mathrm{T}$ cells during the acute phase of the infection.

Severe dengue infection has a tendency of developing haemorrhage involving blood hemolysis [63, 64] and it is known that blood hemolysis contributes to the accumulation of serum ferritin as well as from damaged cells [65]. In addition, activated macrophages also secrete ferritin into plasma although its mechanism is still unknown [66]. Since findings from our study showed that both serum ferritin and soluble CD163 level are correlated to each other in severe dengue patients, we suggest that both markers can be used concurrently to discriminate severe dengue patients from the dengue fever patients. The value of the area under the (ROC) curve (AUCs) is a reflection of the overall ability of the test in discriminating severe dengue patients from dengue patients. ROC is used to determine a cutoff value for a clinical test. Here, we showed that analysis of sCD163 levels of the severe dengue group yielded a fair AUC value of 0.72 when tested against sCD163 from the dengue fever group. Moreover, an excellent AUC value of 1.00 was obtained when the sCD163 levels of severe dengue patients were analysed in combination with patients with ferritin levels above $10000 \mu \mathrm{g} / \mathrm{L}$, supporting the fitness of sCD163 as a differential marker for severe dengue disease. Previously, the use of blood monocyte CD163 as a biomarker for HIV-1 infection and neuroids was reported [67]. sCD163 is potentially an important marker to predict impending severe dengue and until an effective drug or vaccine is available for dengue, a good diagnostic marker of severe dengue is crucial to improve management of severe dengue patients.

Figure 4. Total population of circulating CD163-bearing monocytes in dengue patients. Gating strategy of CD163+ monocytes population originated from total monocytes of peripheral blood (4A). Lower population of CD163+ monocytes is observed in severe dengue (4B) compared to the healthy control group. $* P<0.05, * * P<0.01, * * * P<0.001$. 
In summary, two distinct markers of macrophage activation syndrome were investigated in dengue patients. Hyperferritinemia and increased serum SCD163 are shown in the study to be consistent with severe manifestations of dengue. Ferritin and sCD163, hence, could be charted and noted as important markers for monitoring dengue disease progression.

\section{Acknowledgement}

This study received funding by the Ministry of Higher Education Malaysia via the Long Term Research Grant Scheme (LRGS Grant No. LR001-2011C) and the High Impact Research (HIR)-MOHE Grant (E000013-20001). We thanked Ms. Nurul Izzani Zulkefli, Mdm. Ong Soek Har and Mdm. Indrani Pemmulu for patient recruitment and samples collection and Ms. Shu Meng Hooi for assisting in blood samples processing. We appreciate the kind assistance of Ms. Shu Meng Hooi and Mr. Jefree Johari in ethics application. We also thanked Dr. Sharifah Faridah and Prof. Lucy Lum Chai See for being the attending clinicians and collaborators for this study.

\section{Abbreviations}

MAS: macrophage activation syndrome; sCD163: soluble CD163; sCD25: soluble CD25; mCD163: membrane-bound CD163; ROC: receiver operating curve; AUC: area under curve; ADE: antibody dependent enhancement; DHF: dengue hemorrhagic fever; DSS: dengue shock syndrome; HS: hemophagocytic syndrome; sJIA: systemic juvenile idiopathic arthritis; HIV: human immunodeficiency virus; GCP: good clinical practices; ID: infectious diseases; UMMC: university of Malaya medical center; NS1: non-structural protein 1; WHO: world health organization; SST: serum separator tube; CDC: centers for disease control and prevention; CEIA: chemiluminescence enzyme immunoassay; DENV: dengue virus; $\mathrm{Hb}-\mathrm{Hp}$ : hemoglobin-haptoglobin.

\section{Competing Interests}

The authors have declared that no competing interest exists.

\section{References}

1. AbuBakar S, Shafee N. Outlook of dengue in Malaysia: a century later. Malays J Pathol. 2002; 24: 23-27.

2. Brady OJ, Gething PW, Bhatt S, et al. Refining the global spatial limits of dengue virus transmission by evidence-based consensus. PLoS Negl Trop Dis. 2012; 6: e1760.

3. Duong V, Lambrechts L, Paul RE, et al. Asymptomatic humans transmit dengue virus to mosquitoes. Proc Natl Acad Sci U S A. 2015, 112:14688-93.

4. Ho TS, Wang SM, Anderson R, at al. Antibodies in dengue immunopathogenesis. J Formos Med Assoc. 2013; 112: 1-2

5. de Alwis R, Williams KL, Schmid MA, Lai CY, Patel B, Smith SA. Dengue viruses are enhanced by distinct populations of serotype cross-reactive antibodies in human immune sera. PLoS Pathog. 2014; 10: e1004386.

6. Rothman AL, Francis AE. Immunopathogenesis of dengue haemorrhagic fever. Virol. 1999; 257: 1-6.
7. Rothman AL. Immunity to dengue virus: a tale of original antigenic sin and tropical cytokine storms. Immunol. 2011; 11: 532-543.

8. Sun Y, Jin C, Zhan F, et al. Host cytokine storm is associated with disease severity of severe fever with thrombocytopenia syndrome. J Infect Dis. 2012; 206: 1085-1094.

9. Pang T, Cardosa MJ, Guzman MG. Of cascade and perfect storms: the immunopathogenesis of dengue haemorrhagic fever - dengue shock syndrome (DHF/DSS). Immunol Cell Biol. 2007; 85: 43-45.

10. Malavige GN, Huang LC, Salimi M, et al. Cellular and cytokine correlates of severe dengue infection. PLoS One. 2012; 7: e50387

11. Sam SS, Omar SF, Teoh BT, et al. Review of dengue hemorrhagic fever fatal cases seen among adults: a retrospective study. PLoS Negl Trop Dis. 2013; 7: e2194.

12. Srichaikul T, Punyagupta S, Kanchanapoom T, et al. Hemophgocytic syndrome in dengue hemorrhagic fever with severe multiorgan complications. J Med Assoc Thai. 2008; 91: 104-109.

13. Rueda E, Mendez A, Gonzalez G. Hemophagocytic syndrome associated with dengue hemorrhagic fever. Biomedica. 2002; 22: 160-166.

14. Tan LH., Lum CH., Omar SF, et al.. Hemophagocytosis in dengue: Comprehensive Report of Six Cases. J Clin Virol. 2012; 55: 79-82.

15. Ab-Rahman HA, Wong PF, Rahim H, Ab-Jamil J, Tan KK, Sulaiman S, Lum CS, Syed-Omar SF, AbuBakar S. Dengue death with evidence of hemophagocytic syndrome and dengue virus infection in the bone marrow. SpringerPlus. 2015; 4: 665.

16. Lu PL., Hsiao HH, Tsai JJ., et al. Dengue virus-associated hemophagocytic syndrome and dyserythropoiesis: A case report. Kaohsiung J Med Sci. 2005; 21: 34-39.

17. Ravelli A, Magni-Manzoni S, Pistorio A, et al. Preliminary diagnostic guidelines for macrophage activation syndrome complicating systemic juvenile idiopathic arthritis. J Pediatr. 2005; 146: 598-604.

18. Schaer DJ, Schleiffenbaum B, Kurrer $M$, et al. Soluble haemoglobin-haptoglobin scavenger receptor CD163 as a lineage-specific marker in the reactive hemophagocytic syndrome. Eur J Haem. 2015; 74: 6-10.

19. Bleesing J, Prada A, Siegel DM, et al. The diagnostic significance of soluble CD163 and soluble interleukin-2 receptor a-chain in macrophage activation syndrome and untreated new-onset systemic juvenile idiopathic arthritis. Arthritis rheumatol. 2007; 56: 965-971.

20. Rosario C, Gisele ZD, Esther GMH, et al. The hyperferritinemic syndrome: macrophage activation syndrome, Still's disease, septic shock and catasthropic antiphospholipid syndrome. BioMed Central. 2013; 11: 185.

21. Allen CE, Yu X, Kozinetz CA, et al. Highly elevated ferritin levels and the diagnosis of hemophagocytic lymphohistiocytosis. Pediatr Blood Cancer. 2008; 50: 1227-1235

22. Bennet TD, Fluchel M, Hersh AO, et al. Macrophage activation syndrome in children with systemic lupus erythematosus and children with juvenile idiopathic arthritis. Arthritis Rheum. 2012; 64: 4135-4142.

23. Shimizu M, Yachie A. Compensated inflammation in systemic juvenile idiopathic arthritis: role of alternatively activated macrophages. Cytokine. 2012; 60: $226-232$

24. Minoia F, Davi S, Horne AC, et al. Clinical features, treatment and outcome of macrophage activation syndrome complicating systemic juvenile idiopathic arthritis. Arthritis Rheumatol. 2014; 66: 3160-3169.

25. Ravelli A, Grom AA, Behrens EM, et al. Macrophage activation syndrome as part of systemic juvenile idiopathic arthritis: diagnosis, genetics, pathophysiology and treatment. Genes Immun. 2012; 13: 289-298.

26. Lei HY. Transient hemophagocytic activity in dengue immunopathogenesis. J Formos Med Assoc. 2009; 108: 595-598

27. Ray S, Kundu S, Saha M, et al. Hemophagocytic syndrome in classic dengue fever. J Glob Infect Dis. 2011; 3: 399-401.

28. Chaiyaratana W, Chuansumrit A, Atamasirikul K, et al. Serum ferritin level in children with dengue infection. Southeast Asian J Trop Med Public Health. 2008; 39: 832-836.

29. van de Weg CAM, Huits RMHG, Pannuti CS, et al. Hyperferritenaemia in dengue virus infected patients is associated with immune activation and coagulation disturbances. PLoS Neg Trop Dis. 2014; 8: e3214.

30. Borda JT, Alvarez X, Mohan M, et al. CD163, a marker of perivascular macrophages, is up-regulated by microglia in simian immunodeficiency virus encephalitis after haptoglobin-hemoglobin complex stimulation and is suggestive of breakdown of the blood barrier. Am J Pathol. 2008; 172: 725-737.

31. Kusi KA, Gyan BA, Goka BQ, et al. Level of soluble CD163 and severity of malaria in children in Ghana. Clin Vaccine Immunol. 2008; 15: 1456-1460.

32. Mendonca VRR, Luz NF, Santos NJG, et al. Association between the haptoglobin and heme oxygenase 1 genetic profiles and soluble CD163 in susceptibility to severity of human malaria. Infect Immun. 2012; 80: 1445-1454.

33. Chua CLL, Brown GV, Hamilton JA, et al. Soluble CD163, a product of monocyte/macrophage activation, is inversely associated with haemoglobin levels in placental malaria. PLoS One. 2013; 8: e64127.

34. Shaked I, Hanna DB, Bleibner C, et al. Macrophage inflammatory markers are associated with subclinical carotid artery diseasein woman with human immunodeficiency virus or hepatitis $C$ virus infection. Arterioscler Thromb Vasc Biol 2014; 34:1085-1092.

35. Wang J, Guo W, Du H, et al. Elevated soluble CD163 plasma levels are associated with disease severity in patients with hemorrhagic fever with renal syndrome. PLoS One. 2014; 9: e112127. 
36. Fink K, Zellweger R, Weber J, et al. Long-term maternal imprinting of the specific B cell repertoire by maternal antibodies. Eur J Immunol. 2008; 38:90-101.

37. AbuBakar S, Shu MH, Johari J, et al. Senescence affects endothelial cells susceptibility to dengue virus infection. Int J Med Sci. 2014; 11: 538-544.

38. CDC. Laboratory guidance and diagnostic testing; Revised 18 July 2010. Atlanta, USA: CDC. 2010

39. World Health Organization and the Special Programme for Research and Training in Tropical Diseases (TDR). Dengue guidelines for diagnosis, treatment, prevention and control. Geneva, Switzerland: World Health Organization; 2009.

40. Kurane I, Ennis FA. Immunopathogenesis of dengue virus infections. In: Gubler DJ ed, Kuno G 2nd eds. Dengue and Dengue Haemorrhagic Fever. CAB International: Oxford,1997: 273-290.

41. Nguyen TH, Nguyen TL, Lei HY, et al. Association between sex, nutritional status, severity of dengue hemorrhagic fever, and immune status in infants with dengue hemorrhagic fever. Am J Trop Med Hyg. 2005; 72: 370-374.

42. Chakravarti A, Kumaria R. Circulating levels of tumor necrosis factor-alpha and interferon-gamma in patients with dengue and dengue haemorrhagic fever during an outbreak. Indian J Med Res. 2006; 123: 25-30.

43. Perez AB, Garcia G, Sierra B, et al. IL-10 levels in dengue patients: some findings from the exceptional epidemiological conditions in Cuba. J Med Virol. 2004; 73: 230-234.

44. Priyadarshini D, Gadia RR, Tripathy A, et al. Clinical findings and pro-inflammatory cytokines in dengue patients in Western India: a facility-based study. PlosOne. 2010, 5: e8709.

45. Tuchinda M, Dhorranintra B, Tuchinda P. Histamine content in 24-hour urine in patients with dengue haemorrhagic fever. Southeast Asian J Trop Med Public Health. 1977; 8: 80-83.

46. Phan DT, Ha NT, Thuc LT, et al. Some changes inimmunity and blood in relation to clinical states of dengue hemorrhagic fever patients in Vietnam. Haematologia (Budap). 1991; 24: 13-21.

47. AbuBakar S, Azmi A, Mohamed-Saad N, et al. Antibody responses of dengue fever patients to dengue 2 (New Guinea C strain) viral proteins. Malaysian J Pathol. 1997; 19: 41-51.

48. Bozza FA, Cruz OG, Zagne SM, et al. Multiplex cytokine profile from dengue patients: MIP-1 $\beta$ and IFN- $\gamma$ as predictive factors for severity. BMC Infect Dis. 2008; 8: 86.

49. Mustafa AS, Elbishbishi EA, Agarwal R, et al. Elevated levels of interleukin-13 and IL-18 in patients with dengue hemorrhagic fever. FEMS Immunol Med Microbiol. 2001; 30:229-233.

50. Colafrancesco S, Priori R, Alessandri C, et al. The hyperferritinemic syndrome and CD163: a marker of macrophage activation syndrome. Isr Med Assoc J. 2014; 16: 662-663.

51. Buechler C, Ritter M, Orso E, et al. Regulation of scavenger receptor CD163 expression in human monocytes and macrophages by pro- and anti-inflammatory stimuli. J Leukoc Biol. 2000; 67: 97-103.

52. Abraham NG, Drummond G. CD163-mediated haemoglobin-heme uptake activates macrophage $\mathrm{HO}-1$, providing an anti-inflammatory function. Circ Res. 2006; 99: 911-914.

53. Onofre G, Kolackova M, Jankovicova K, et al. Scavenger receptor CD163 and its biological functions. Acta Medica (Hradec Kralove). 2009; 52: 57-61.

54. Suharti C, Van Gorp ECM, Dolmans WMV, et al. Cytokine patterns during dengue shock syndrome. Eur Cytokine Netw. 2003; 14: 172-177.

55. Tsai TT, Chuang YJ, Lin YS, et al. An emerging role for the anti-inflammatory cytokine interleukine-10 in dengue virus infection. J Biomed Sci. 2013; 20:40.

56. Moestrup SK, Moller HJ. CD163: A regulated haemoglobin scavenger receptor with a role in anti-inflammatory response. Ann Med. 2004; 36: 347-354.

57. Fabriek BO, Dijkstra CD, van den Berg TK. The macrophage scavenger receptor CD163. Immunobiology. 2005; 210: 153-160.

58. Kowal K, Silver R, Slawinska E, et al. CD163 and its role in inflammation. Folia Histochem Cytobiol. 2011; 49: 365-374

59. Akila $P$, Prashant V, Suma MN, et al. CD163 and its expending repertoire. Clin Chim Acta. 2012; 413: 669-674.

60. Davis BH, Zarev PV. Human monocyte CD163 expression inversely correlates with soluble CD163 plasma level. Cytometry B:Clin Cytom. 2005; 63: 16-22.

61. Ingels C, Moller HJ, Hansen TK, et al. Circulating levels of the shed scavenger receptorsCD163 and association with outcome of critically ill patients. J Clin Immunol. 2013; 33: 619-629.

62. Strauss R, Neureiter D, Westenburger B, et al. Multifactorial risk analysis of bone marrow histiocytic hyperplasia with hemophagocytosis in critically ill medical patients - a post-mortem clinicopathologic analysis. Crit Care Med. 2004; 32: 1316-1321.

63. Khan S, Gupta ND, Maheshwari S. Acute gingival bleeding as a complication of dengue hemorrhagic fever. J Indian Soc Periodontol. 2013; 17: 520-522.

64. Zhang FC, Zhao H, Li LH, et al. Severe dengue outbreak in Yunan, China, 2013. Int J Infect Dis. 2014; 27: 4-6.

65. Kell DB, Pretorius E. Serum ferritin is an important inflammatory disease marker, as it is mainly a leakage product from damaged cells. Metallomics. 2014; 6: 748-773.

66. Orino K, Watanabe K. Molecular, physiological and clinical aspects of the iron storage protein ferritin. Vet J. 2008; 178: 191-201.

67. Rappaport J, Fischer-Smith T. Blood monocyte CD163 expression as a biomarker in HIV-1 infection and neuroids. United States patent US 8,273,538 B2. 2012 Sept 25 . 